\title{
Ti-based BCC Alloy: Dehydrogenation Characterization Using Synchrotron and Neutron Diffraction
}

\author{
Thomas Bibienne ${ }^{a}$, Roxana Flacau ${ }^{b}$, Chang-Yong Kim ${ }^{c}$, Jean-Louis Bobet ${ }^{d}$, Jacques Huot ${ }^{a}$ * \\ a'Institut de Recherche sur l'Hydrogène, Université du Québec à Trois-Rivières, 3351, Boul. des Forges, \\ Trois-Rivières, Québec, G9A 5H7, Canada \\ ${ }^{b}$ Canadian Neutron Beam Centre, National Research Council Canada, Chalk River Laboratories, \\ Building 459, Chalk River, Ontario, KOJ 1J0, Canada \\ ${ }^{c}$ Canadian Light Source Inc., 44 Innovation Boulevard, Saskatoon, Saskatchewan, S7N 2V3, Canada \\ ${ }^{d}$ Institut de Chimie de la Matière Condensée de Bordeaux, Université de Bordeaux, 87 Avenue du Dr. \\ Schweitzer, 33600, Pessac, France
}

Received: January 28, 2016; Revised: April 17, 2016; Accepted: April 27, 2016

\begin{abstract}
Dehydrogenation of a BCC alloy of composition 52Ti-12V-36Cr doped with 4 wt. $\% \mathrm{Zr}_{7} \mathrm{Ni}_{10}$ was studied by diffraction using synchrotron and neutron radiations. In the case of synchrotron radiation dehydrogenation was imposed by raising the temperature while keeping the sample under vacuum. We found that prior to final dehydrogenation the FCC dihydride phase is becoming metastable. Neutron in-situ experiment was done by keeping the deuterided sample at $200^{\circ} \mathrm{C}$ and slowly reducing the pressure. This lead to a reduction of the occupation factor of deuterium in the FCC phase and an increase of the isotropic atomic temperature factor of deuterium.
\end{abstract}

Keywords: $B C C$ alloys, in-situ measurements, synchrotron radiation, neutron diffraction

\section{Introduction}

For the development of hydrogen as an energy carrier, there is a strong need for a safe, compact, and low cost means to store hydrogen. Metal hydrides are ideal candidates for storing hydrogen because they have high volumetric capacities and could operate under relatively low pressure at room temperature. Titanium containing body centered cubic (BCC) alloys are considered to be good candidates for room temperature applications ${ }^{1-3}$. In a recent series of papers we reported the investigation of two T-V-Cr BCC alloys doped with 4 wt. $\%$ of $\mathrm{Zr}_{7} \mathrm{Ni}_{10}$ alloy: $52 \mathrm{Ti}-12 \mathrm{~V}-36 \mathrm{Cr}$ and $42 \mathrm{Ti}-21 \mathrm{~V}-37 \mathrm{Cr}^{4,5}$. In these investigations we used two ways to synthesize the compounds: i) first melt the $\mathrm{Zr}_{7} \mathrm{Ni}_{10}$ and $\mathrm{Ti}-\mathrm{V}-\mathrm{Cr} \mathrm{BCC}$ alloys separately, mix them in the right proportion and thereafter melt the mixture; ii) mix all elements in the desired stoichiometry and perform a single melt. However, irrespective of the synthesis method, the microstructure consisted of a matrix and an intergranular phase. The matrix was made of Ti- $\mathrm{V}-\mathrm{Cr}$ with small amount of $\mathrm{Zr}$ and $\mathrm{Ni}$. The intergranular phase was is also made of Ti-V-Cr-Zr-Ni atoms but, compared to the matrix stoichiometry, depleted in vanadium and chromium and enriched in nickel and zirconium. The main effect of doping Ti-V-Cr BCC alloys with $\mathrm{Zr}_{7} \mathrm{Ni}_{10}$ was that the alloys presented a quick first hydrogenation without prior heat treatment. We found that, even if the microstructure was slightly different, the hydrogen sorption behaviour alloys synthesized by both methods was analogous, and their thermodynamic parameters very close. As single melt process is much easier than co-melt we decided to investigate further the alloys made by single melt method. In order to have a better understanding of the dehydrogenation process in these compounds, we performed in-situ experiments using neutron and synchrotron radiations.

* e-mail: jacques.huot@uqtr.ca

\section{Experimental details}

All alloys were prepared by using raw elements from Alfa-Aesar which had the following purities: Ti (99.95\%), $\mathrm{V}$ (99\%), Cr (99\%), Zr (99.95\%) and $\mathrm{Ni}(99.7 \%)$. The composition $52 \mathrm{Ti}-12 \mathrm{~V}-36 \mathrm{Cr}$ doped with 4 wt. $\%$ of $\mathrm{Zr}_{7} \mathrm{Ni}_{10}$ was synthesized which correspond to a bulk nominal value 50.6Ti-11.7V-35Cr-1.1Zr-1.6Ni. The casting was performed using the single melt process, where all raw components were melted together in an arc furnace. In casting, each sample was turned over and melted three times to ensure homogeneity.

The microstructure was investigated with a Tescan Vega3 SEM. An Electron Probe Micro Analyses (EPMA), coupled with Wavelength Dispersive (WDS), was used for elements mapping.

The synchrotron $\mathrm{x}$-ray scattering experiments were carried out at the Hard X-ray MicroAnalysis (HXMA) beamline at the Canadian Light Source (CLS) using X-ray energy of the $\mathrm{Zr}$ K-edge (wavelength $0.06884 \mathrm{~nm}$ ). The sample was inserted into a quartz capillary of outside diameter $1.0 \mathrm{~mm}$ and wall thickness $0.01 \mathrm{~mm}$. For desorption the capillary was continuously pumped with a mechanical pump. Two heating coils near the capillary were used to increase the temperature. Integration of 2-D data to 1-D data was performed using Fit2d software ${ }^{6}$.

Neutron powder diffraction (NPD) experiments were carried out on the $\mathrm{C} 2$ high-resolution neutron powder diffractometer at the NRU reactor at the Chalk River Laboratories. The instrument is equipped with an 800 -wire position-sensitive detector covering a range of $80^{\circ}$. Neutron wavelength of $0.13287 \mathrm{~nm}$ was selected using an $\mathrm{Si}(531)$ monochromator. NPD patterns of as-cast and desorbed samples were registered. Also, in situ desorption neutron diffraction were recorded. In this setup, the hydride was kept under deuterium pressure (to prevent it to desorb) and the temperature was raised up. 
Then, the hydrogen pressure was decreased step by step and NPD patterns were recorded. The crystallographic parameters (lattice parameters, crystallite size, microstrain, occupation factors and atomic temperature factors) were evaluated by Rietveld refinement of the X-ray and neutron powder diffraction patterns using Topas software?

\section{Results and discussion}

\subsection{Microstructure}

The microstructure of the as-cast alloy $52 \mathrm{Ti}-12 \mathrm{~V}$ $36 \mathrm{Cr}+4 \%$ wt. $\mathrm{Zr}_{7} \mathrm{Ni}_{10}$ is shown in figure 1 . The alloy is made up of a matrix (grey phase) and a secondary phase (bright areas). From electron probe micro analysis (EPMA) the chemical composition of the matrix and the one of the intergranular phase were measured to be respectively 51.2Ti-11.8V-34.8Cr-0.8Zr-1.4Ni and 50.3Ti-7.7V-30Cr$7 \mathrm{Zr}-5 \mathrm{Ni}$. A more complete discussion of the microstructure and respective chemical composition of both phases could be found in ref 5 .

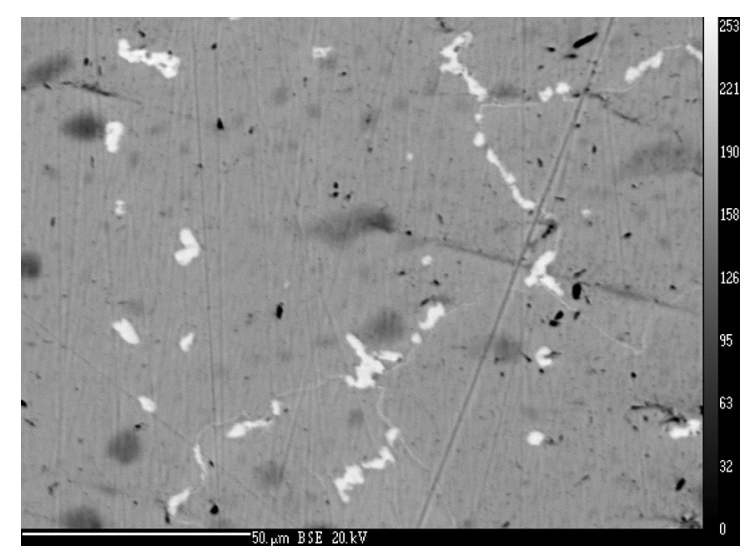

Figure 1: Backscattering electron micrograph of as-cast $52 \mathrm{Ti}-12 \mathrm{~V}$ $36 \mathrm{Cr}+4 \%$ wt. $\mathrm{Zr}_{7} \mathrm{Ni}_{10}$.

\subsection{Synchrotron radiation diffraction patterns}

In situ desorption synchrotron analysis was performed on a hydrided sample. Dehydrogenation was performed by heating the sample at $2{ }^{\circ} \mathrm{C} / \mathrm{min}$ while continuously pumping. Figure 2 shows the diffraction patterns taken at regular intervals during heating. The initial pattern taken at $25^{\circ} \mathrm{C}$ present two phases: the FCC (Face Centered Cubic, S. G. $\mathrm{Fm} 3 \mathrm{~m}$ ) dihydride phase and a BCT monohydride phase (Body Centered Tetragonal, S.G. $14 / \mathrm{mmm}$ ). Details of the FCC and BCT phases are given in the paper by Nakamura et al. ${ }^{8}$.

In the present case, it should be pointed out that the lattices parameters of the BCT phase fitting the pattern at $25^{\circ} \mathrm{C}$ are very close to each other. Actually, for all patterns, fitting with the monohydride BCT phase just gave a slightly better fit than fitting with the fully dehydrided BCC phase (Body Centered Cubic, S.G. Im $3 m$ ). The patterns were fitted using the BCT phase mainly for thermodynamic reasons as the dihydride should desorbs to the monohydride ${ }^{8}$. But from the diffraction patterns

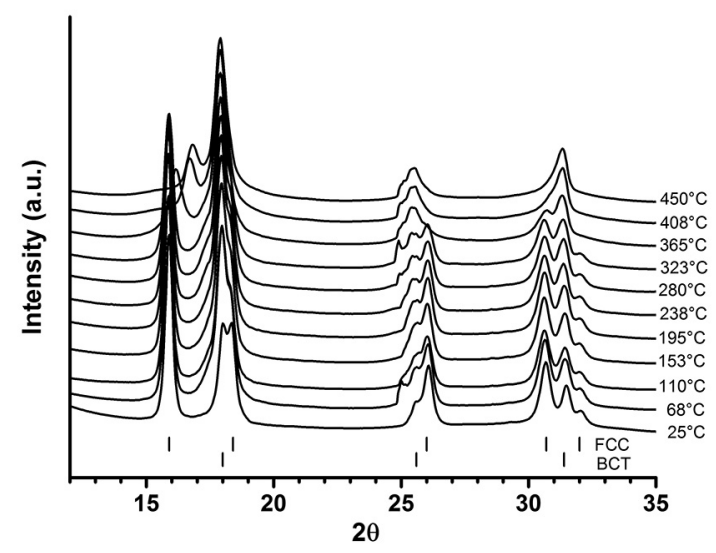

Figure 2: Synchrotron radiation diffraction patterns of hydrided 52Ti-12V-36Cr+4\%wt. $\mathrm{Zr}_{7} \mathrm{Ni}_{10}$.

alone the BCT phase could not be distinguish from the BCC phase therefore, we could not claim with $100 \%$ certitude that the BCT phase is present instead of a BCC phase. Assuming a BCT phase, from Rietveld refinement we found that, at $25^{\circ} \mathrm{C}, 65 \mathrm{wt} . \%$ of the sample is made of FCC phase and 35 wt. $\%$ of BCT.

Figure 2 shows that upon heating up to $365^{\circ} \mathrm{C}$ the phase abundances of FCC and BCT slightly changed. From Rietveld refinement the phase abundance of FCC went from $65(8)$ wt. $\%$ to $44(8)$ wt. $\%$ when the temperature rose from $25^{\circ} \mathrm{C}$ to $323^{\circ} \mathrm{C}$. At the same time, the lattice parameter of the FCC phase was essentially constant but in the case of the BCT phase the lattice parameters slightly increased (see table 1). At $365^{\circ} \mathrm{C}$, when the sample was dehydriding, the $a$ and $c$ lattice parameters of the BCT phase were much bigger compared to $25^{\circ} \mathrm{C}$. In the pattern at $450^{\circ} \mathrm{C}$ the amount of FCC was down to $13 \mathrm{wt} . \%$. We see that at $450^{\circ} \mathrm{C}$ the $\mathrm{BCT}$ lattices were essentially the same thus, we could consider it to be a BCC phase which means that the BCT totally desorbed. In the case of the FCC phase we see that between $25^{\circ} \mathrm{C}$ and $323^{\circ} \mathrm{C}$ the (111) peak is barely moving but that at higher temperature it moves toward higher angle, reaching $17^{\circ}$ at $450^{\circ} \mathrm{C}$. This means that the lattice parameter is greatly reduced as confirmed by the Rietveld refinement reported in Table 1. Also, the relative intensities of the Bragg peaks changed. We could interpret this as the FCC being unstable because it was far from its equilibrium state. The fact that the FCC and BCC phase coexists and there is no BCT phase is thermodynamically impossible. However, we think that in this case the system was kinetically controlled. It means that the dehydrogenation of the FCC phase was very slow compared to the temperature increase and thus the system was 'lagging behind' from a thermodynamic point of view. Practically, it means that the dehydrogenation of the FCC phase is much slower than the dehydrogenation of the BCT phase. At temperatures above $400^{\circ} \mathrm{C}$ as soon as the FCC phase goes down to the BCT phase the later desorb to the BCC phase. The whole process of desorption from the FCC to the BCC phase is kinetically controlled at temperature above $400^{\circ} \mathrm{C}$. 
Table 1: Lattice parameters of the BCT and FCC phases in 52Ti$12 \mathrm{~V}-36 \mathrm{Cr}+4 \%$ wt. $\mathrm{Zr}_{7} \mathrm{Ni}_{10}$ alloy in function of temperature as deduced from Rietveld refinement of the synchrotron radiation patterns. Numbers in parenthesis are one standard deviation of the last significant digit.

\begin{tabular}{lccc}
\hline \multirow{2}{*}{$\begin{array}{l}\text { Temperature } \\
\left({ }^{\circ} \mathrm{C}\right)\end{array}$} & \multicolumn{2}{c}{$\mathrm{BCT}$} & FCC \\
\cline { 2 - 4 } & $a(\mathrm{~nm})$ & $c(\mathrm{~nm})$ & $a(\mathrm{~nm})$ \\
\hline 25 & $0.311(1)$ & $0.311(2)$ & $0.43204(8)$ \\
323 & $0.3146(5)$ & $0.3129(9)$ & $0.4336(2)$ \\
365 & $0.318(3)$ & $0.317(6)$ & $0.4321(6)$ \\
450 & $0.313(3)$ & $0.313(2)$ & $0.410(2)$ \\
\hline
\end{tabular}

\subsection{Neutron diffraction}

Neutron diffraction of $52 \mathrm{Ti}-12 \mathrm{~V}-36 \mathrm{Cr}+4 \%$ wt. $\mathrm{Zr}_{7} \mathrm{Ni}_{10}$ presents a special peculiarity. As measured by EDX, the composition of the matrix and the one of the intergranular phase are respectively $51.2 \mathrm{Ti}-11.8 \mathrm{~V}-34.8 \mathrm{Cr}-0.8 \mathrm{Zr}-1.4 \mathrm{Ni}$ and 50.3 Ti-7.7V-30Cr-7Zr-5Ni. For neutron diffraction, the scattered intensity is proportional to the weighted average of the coherent scattering length of the elements. In the present case, from the published values for $\mathrm{Ti}, \mathrm{V}, \mathrm{Cr}, \mathrm{Zr}$, and $\mathrm{Ni}$ scattering the average scattering length of the matrix and the intergranular phase are respectively $-0.3389 \mathrm{fm}$ and $0.3479 \mathrm{fm}^{9}$. In absolute value, these two numbers are smaller than the scattering length of vanadium $(-0.3824 \mathrm{fm})$. It is well known that vanadium is 'transparent' to thermal neutron. This is the reason why our sample cell was made of vanadium. Therefore, the $52 \mathrm{Ti}-12 \mathrm{~V}-36 \mathrm{Cr}+4 \% \mathrm{wt}$. $\mathrm{Zr}_{7} \mathrm{Ni}_{10}$ alloy is in fact a so-called 'null matrix' alloy and do not present any Bragg peaks in neutron diffraction. This was confirmed in the present experiment but, as there is no information to be taken from these patterns, the neutron powder diffractions (NPD) of the as-cast and desorbed samples are not presented here. However, a small amount of deuterium will give rise to Bragg's peaks. Thus, doing in-situ diffraction could give us information about the localisation and abundance of deuterium in the crystal structure.

Because of the 'null matrix' nature of this alloy, special care should be taken in the Rietveld refinement. As the scattering length of metal atoms adds up to almost zero, refining the occupation factor and the corresponding isotropic atomic temperature factors $\left(\mathrm{B}_{\text {iso }}\right)$ could easily leads to unrealistic values of these parameters. Therefore, the occupation factor of the metallic atoms was fixed to the nominal values. In order to use a realistic value of $\mathrm{B}_{\text {iso }}$ we used the compilation made by Peng et al. ${ }^{10}$. This compilation is only for pure elements but it should give us the right order of magnitude for the $\mathrm{B}_{\text {iso }}$. At $27^{\circ} \mathrm{C}$ the $\mathrm{B}_{\text {iso }}$ values for Ti(HCP), V(BCC), and $\mathrm{Cr}(\mathrm{BCC})$ are respectively $0.5261 \AA^{2}, 0.5855 \AA^{2}$, and $0.2569 \AA^{2}$. As the $B_{\text {iso }}$ value also depends on the crystal structure we wanted to compare with a transition metal that has a FCC phase at room temperature. In its FCC phase, iron has a $\mathrm{B}_{\text {iso }}$ of $0.5710 \AA^{2}$ which is much higher than the value of $0.3328 \AA^{2}$ for $\mathrm{Fe}(\mathrm{BCC})$. Thus, we are confident that $\mathrm{a} \mathrm{B}_{\text {iso }}$ value of $0.5 \AA^{2}$ could be assigned to the metal atoms in the present FCC phase.

In the Rietveld refinements of neutron diffraction patterns only the following crystal parameters were refined: Lattice parameter, crystallite size, microstrain, atomic occupation factor and $\mathrm{B}_{\text {iso }}$ of deuterium atom. As the average scattering length of the metal atoms is near zero, the occupation factor was fixed to their nominal values and only the occupation factor of deuterium was refined.

In the in-situ experiment the sample was first exposed to $500 \mathrm{kPa}$ of deuterium at room temperature. Figure 3 presents the neutron powder diffraction pattern of the deuterated sample along with the corresponding Rietveld refinement. One can clearly see the diffraction peaks of the dihydride FCC phase. Small peaks can be observed between $2 \theta=37^{\circ}$ and $47^{\circ}$ but the intensities are too small to conclude with certainty on the nature of these peaks and we could not conclude that they belong to the intergranular phase. As explained previously, the bulk composition of this alloy give a neutron scattering length very close to zero. Therefore, it is not surprising to see that the intergranular phase could not be seen unless it absorbs deuterium.

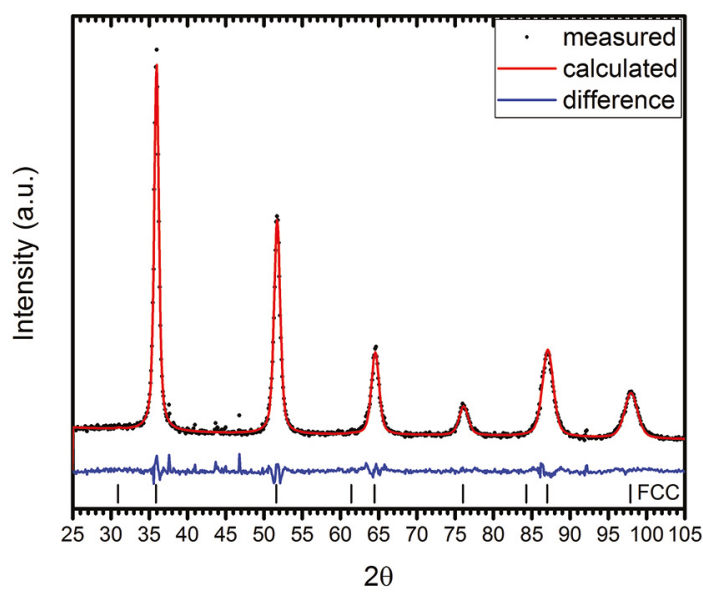

Figure 3: Neutron powder diffraction patterns of hydrided 52Ti-12V-36Cr+4\%wt. $\mathrm{Zr}_{7} \mathrm{Ni}_{10}$.

As the non-deuterided phases do not show any Bragg peaks the only way to verify that the sample is hydrided is to measure the occupation factor of the deuterium site. In the FCC phase hydrogen is located in a tetragonal site (Wickoff position $8 c(1 / 4,1 / 4,1 / 4))$.

Figure 3 shows that the Rietveld refinement is quite good. The different R-values are displayed in Table 2 and the values of the refined crystal parameters are presented in Table 3. We see that the sample is not fully hydride as the occupation factor is only 0.62 which translate to a $\mathrm{Ti}_{0.506} \mathrm{~V}_{0.117} \mathrm{Cr}_{0.35} \mathrm{Zr}_{0.011} \mathrm{Ni}_{0.016} \mathrm{D}_{1.24}$ composition. The reason why a full deuteration was not achieved may be because of the slow absorption kinetics at $25^{\circ} \mathrm{C}$.

Table 2: R-values of Rietveld refinement displayed in Figure 3. GOF: Goodness of fit; $\mathrm{R}_{\text {exp }}$ : expected weighted profile R-factor; $\mathrm{R}_{\mathrm{wp}}$ : weighted profile R-factor; DW: weighted Durbin-Watson.

\begin{tabular}{llll}
\hline GOF & $\mathrm{R}_{\text {exp }}$ & $\mathrm{R}_{\text {wp }}$ & $\mathrm{DW}$ \\
\hline 1.81 & 2.25 & 4.06 & 1.13 \\
\hline
\end{tabular}

For the in-situ experiment, the temperature was raised to $200^{\circ} \mathrm{C}$ while keeping the pressure in the vessel at 500 
Table 3: Crystallographic parameters of the FCC phase as evaluated from Rietveld refinement during dehydrogenation of 52Ti-12V$36 \mathrm{Cr}+4 \%$ wt. $\mathrm{Zr}_{7} \mathrm{Ni}_{10}$ alloy. All measurements are at $200^{\circ} \mathrm{C}$ unles stated otherwise. Numbers in parenthesis are one standard deviation of the last significant digit.

\begin{tabular}{|c|c|c|c|c|c|}
\hline \multirow{2}{*}{ Pressure $(\mathrm{kPa})$} & \multirow{2}{*}{$\begin{array}{l}\text { Lattice parameter } \\
\qquad(\mathrm{nm})\end{array}$} & \multirow{2}{*}{$\begin{array}{c}\text { Crystallite size } \\
(\mathrm{nm})\end{array}$} & \multirow{2}{*}{$\begin{array}{c}\text { Microstrain } \\
(\%)\end{array}$} & \multicolumn{2}{|c|}{ Deuterium site } \\
\hline & & & & Occupation factor & $B_{i s o}\left(\AA^{2}\right)$ \\
\hline $500\left(\right.$ at $\left.25^{\circ} \mathrm{C}\right)$ & $0.43125(4)$ & $13.4(3)$ & $0.331(6)$ & $0.62(3)$ & $1.74(2)$ \\
\hline 500 & $0.43202(4)$ & $13.5(3)$ & $0.325(3)$ & $0.84(6)$ & $2.18(3)$ \\
\hline 200 & $0.4308(9)$ & $12.2(3)$ & $0.21(1)$ & $0.60(4)$ & $2.24(3)$ \\
\hline 100 & $0.4297(1)$ & $6.9(2)$ & $0.15(3)$ & $0.53(3)$ & $2.54(7)$ \\
\hline 90 & $0.4296(1)$ & $5.8(2)$ & $0.10(6)$ & $0.39(4)$ & $2.7(1)$ \\
\hline 70 & $0.4288(2)$ & $3.9(1)$ & -- & $0.33(3)$ & $2.8(1)$ \\
\hline 50 & $0.4296(4)$ & $2.3(1)$ & -- & $0.23(2)$ & $2.9(1)$ \\
\hline
\end{tabular}

$\mathrm{kPa}$ of deuterium. This prevented dehydrogenation during heating. The pattern taken at $200^{\circ} \mathrm{C}$ under $500 \mathrm{kPa}$ is shown in figure 4 and the refined parameters in Table 3.

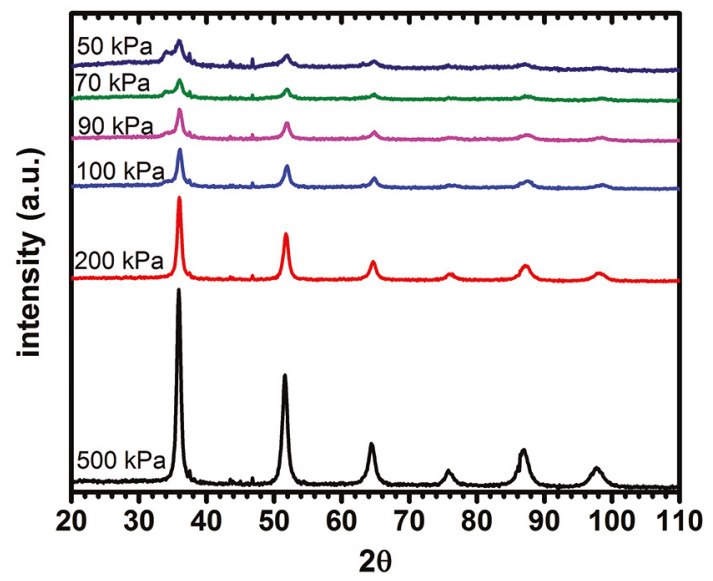

Figure 4: In situ desorption neutron diffraction of the deuterated single-melt $52 \mathrm{Ti}-12 \mathrm{~V}-36 \mathrm{Cr}+4 \% \mathrm{wt}$. $\mathrm{Zr}_{7} \mathrm{Ni}_{10}$ alloy. All diffraction patterns were recorded at $200^{\circ} \mathrm{C}$.

For the Rietveld refinement at $200^{\circ} \mathrm{C}$ the isotropic atomic temperature factor $\mathrm{B}_{\text {iso }}$ of metal atoms was also kept fixed. Again, Peng et al. compilation indicated that Ti(HCP), $\mathrm{V}(\mathrm{BCC})$, and $\mathrm{Fe}(\mathrm{FCC})$ have a $\mathrm{B}_{\text {iso }}$ of respectively 0.8757 $\AA^{2}, 0.9744 \AA^{2}$, and $0.9501 \AA^{2}$. Therefore, a B iso value of 1.0 $\AA^{2}$ was assigned to the metal atoms in the deuterided FCC phase. As for the pattern at $25^{\circ} \mathrm{C}$, only occupation factor and $\mathrm{B}_{\text {iso }}$ of deuterium were refined.

First, comparing the refined values for the patterns at $500 \mathrm{kPa}$ at $25^{\circ} \mathrm{C}$ and $200^{\circ} \mathrm{C}$ we see that the lattice parameter slightly increased with temperature which is what was expected. The crystallite size and microstrain are constant within the experimental errors but the biggest change was observed for the deuterium occupation factor which increased. This means that the sample, which was not fully deuterated at $25^{\circ} \mathrm{C}$, continued to absorbs deuterium as the temperature raised. Because at $200^{\circ} \mathrm{C}$ the plateau pressure is still lower than $500 \mathrm{kPa}$, the sample was always under absorption conditions and thus could continue to absorb deuterium ${ }^{5}$. It should be pointed out that we have measured the plateau pressures using deuterium gas and they are identical to the ones measured under hydrogen and reported in a previous publication $^{5}$.

The other important change is seen for the isotropic atomic temperature factor which increased from $1.75 \AA^{2}$ to $2.18 \AA^{2}$ when the temperature went from $25^{\circ} \mathrm{C}$ to $200^{\circ} \mathrm{C}$. This is also expected as the deuterium should have stronger thermal agitation on its site at higher temperature. From the $\mathrm{B}_{\text {iso }}$ one could find the mean square amplitude of atomic vibration through the relationship ${ }^{11}$.

$$
B_{\text {iso }}=8 \pi^{2}\left\langle r^{2}\right\rangle
$$

Where $\left\langle r^{2}\right\rangle$ is the mean square amplitude of atomic vibration. According to Westlake, a hole should have a radius of at least $0.4 \AA$ in order to be able to accommodate a hydrogen atoms ${ }^{12}$. From the values of $\mathrm{B}_{\text {iso }}$ we found that $\sqrt{ }\left\langle r^{2}\right\rangle$ is $0.148 \AA$ at $25^{\circ} \mathrm{C}$ and $0.166 \AA$ at $200^{\circ} \mathrm{C}$ which are reasonable values for a deuterium atom trapped in a hole.

The occupation factor of deuterium at $200^{\circ} \mathrm{C}$ is very close to the one obtained by Nakamura et al. for their FCC dihydride phase of $\mathrm{Ti}{ }_{0.33} \mathrm{~V}_{0.37} \mathrm{Mn}_{0.3}{ }^{8}$. Therefore, we could consider that the alloy was fully deuterated. The sample was then desorbed by decreasing the deuterium pressure step by step with diffraction patterns recorded at each step. The recorded patterns are presented in Figure 4. One can see that the relative intensity of the peaks decreases as the pressure decreases. For a pressure of $100 \mathrm{kPa}$ a small shoulder appears on the lower angle side of the peak at $36^{\circ}$ and grows as the pressure is lowered. The nature of this shoulder is unknown. A pattern taken at $10 \mathrm{kPa}$ did not show any Bragg peaks except this broad shoulder.

Close inspection of Table 3 reveals the following facts: As the pressure is reduced the sample desorbs deuterium as could be seen by the reduction of occupation factor. Simultaneously, the $\mathrm{B}_{\text {iso }}$ increases with reduction of pressure. The reason of this reduction of $\mathrm{B}_{\text {iso }}$ is not clear but it may be due to the fact that as the number of deuterium atoms diminishes there are less deuterium in the neighborhood of a particular deuterium atom and thus the D-D interaction get weaker thus enabling the deuterium atom to vibrate a little more within its site. Table 3 also shows that during dehydrogenation there is a constant decrease for the crystallite size and microstrain. However, the lattice parameter do not seems to change very much during dehydrogenation, 
contrary to the case of synchrotron radiation. Thus, it seems that the desorption mechanism may be different when it is induced by a temperature increase or by a pressure change. This discrepancy between desorption behavior as seen by synchrotron and neutron may be explained by the details of each experiment. In the case of synchrotron measurement, the temperature was increased relatively quickly $\left(2^{\circ} \mathrm{C} / \mathrm{min}\right)$ and thus the system may at one point be in a metastable phase as we see above $400^{\circ} \mathrm{C}$. On the contrary, the neutron experiment was done at a much slower speed, taking many hours of equilibrium before taking a pattern. Thus the FCC was not metastable phase in this case.

\section{Conclusions}

In this paper we reported the analysis of desorption process in $52 \mathrm{Ti}-12 \mathrm{~V}-36 \mathrm{Cr}$ doped with 4 wt. $\% \mathrm{Zr}_{7} \mathrm{Ni}_{10}$. Two in-situ diffraction experiments were performed: one using synchrotron radiation where a hydrided sample was heated under vacuum, the other using neutron radiation where the deuterated sample was made to release its deuterium by reducing the applied pressure. For both cases the dihydride FCC phase was monitored. We found that in the synchrotron experiment the FCC dihydride phase desorbed to a BCT monohydride phase but as the temperature increases, the FCC becomes metastable with an important reduction of its lattice parameter. At above $400^{\circ} \mathrm{C}$ the kinetics of dihydride $\rightarrow$ monohydride $(\mathrm{FCC} \rightarrow \mathrm{BCT})$ transformation seems to be much slower than the monohydride $\rightarrow$ dehydrided (BCT $\rightarrow$ BCC) reaction and thus the $\mathrm{FCC}$ phase was seen to coexist with the BCC phase.

From in-situ neutron diffraction we were able to precisely measure the occupation factor of deuterium and observe that the isotropic atomic temperature factor increases when the occupation factor decreases. However, we did not see any evidence of the FCC phase being metastable because the experiment was done in a much longer time-scale than the synchrotron one.

\section{Acknowledgements}

This work was supported in part by a Discovery grant from the Natural Sciences and Engineering Research Council of Canada (NSERC). T.B would like to thanks the Canadian Light Source (CLS) for a traveling grant.

\section{References}

1. Sakintuna B, Lamari-Darkrim F, Hirscher M. Metal hydride materials for solid hydrogen storage: A review. International Journal of Hydrogen Energy. 2007;32(9):1121-1140.

2. Akiba E, Iba H. Hydrogen absorption by Laves phase related BCC solid solution. Intermetallics. 1998;6(6):461-470. DOI: http://dx.doi.org/10.1016/S0966-9795(97)00088-5

3. Kabutomori T, Takeda H, Wakisada Y, Ohnishi K. Hydrogen absorption properties of Ti-Cr-A $(\mathrm{A}=\mathrm{V}, \mathrm{Mo}$ or other transition metal) B.C.C. solid solution alloys. Journal of Alloys and Compounds. 1995;231(1-2):528-532. DOI: http://dx.doi. org/10.1016/0925-8388(95)01859-X

4. Bibienne T, Razafindramanana V, Bobet JL, Huot J. Synthesis, characterization and hydrogen sorption properties of a Body Centered Cubic 42Ti-21V-37Cr alloy doped with $\mathrm{Zr}_{7} \mathrm{Ni}_{10}$. Journal of Alloys and Compounds. 2015;620:101-108. DOI: http://dx.doi.org/10.1016/j.jallcom.2014.08.156

5. Bibienne T, Bobet JL, Huot J. Crystal structure and hydrogen storage properties of body centered cubic $52 \mathrm{Ti}-12 \mathrm{~V}-36 \mathrm{Cr}$ alloy doped with $\mathrm{Zr}_{7} \mathrm{Ni}_{10}$. Journal of Alloys and Compounds. 2014;607:251257. DOI: http://dx.doi.org/10.1016/j.jallcom.2014.04.062

6. Hammersley AP, Svensson SO, Hanfland M, Fitch AN, Hausermann D. Two-dimensional detector software: From real detector to idealised image or two-theta scan. High Pressure Research. 1996;14(4-6):235-248. DOI: http://dx.doi. org/10.1080/08957959608201408

7. Bruker-AXS. TOPAS V4: General profile and structure analysis software for powder diffraction data. User's manual. Karlsruhe: Bruker-AXS; 2008.

8. Nakamura Y, Oikawa K, Kamiyama T, Akiba E. Crystal structure of two hydrides formed from a Ti-V-Mn BCC solid solution alloy studied by time-of-flight neutron powder diffraction - a $\mathrm{NaCl}$ structure and a CaF2 structure. Journal of Alloys and Compounds. 2001;316(1-2):284-289. DOI: http://dx.doi. org/10.1016/S0925-8388(00)01503-6

9. Sears VF. Neutron scattering lengths and cross sections. Neutron News. 1992;3(3):26-37. DOI: http://dx.doi. org/10.1080/10448639208218770

10. Peng LM, Ren G, Dudarev SL, Whelan MJ. Debye-Waller Factors and Absorptive Scattering Factors ff Elemental Crystals. Acta Crystallographica Section A: Foundations of Crystallography. 1996;52(3):456-470.

11. Giacovazzo C. The diffraction of X-rays by crystals. In: Giacovazzo C, Ed. Fundamentals of crystallography. Oxford: International Union of Crystallography texts on crystallography/ Oxford University Press; 1992.

12. Westlake DG. Site occupancies and stoichiometries in hydrides of intermetallic compounds: Geometric considerations. Journal of the Less-Common Metals. 1983;90(2):251-273. DOI: http:// dx.doi.org/10.1016/0022-5088(83)90075-9 